\title{
Scanning Methodology for Deployment of Femtocell as Secondary Network
}

\author{
Nikhil P. Wyawahare ${ }^{1^{*}}$ and Sampada Wazalwar ${ }^{2}$ \\ ${ }^{1}$ Assistant Professor Department of Electronics Engineering, \\ ${ }^{2}$ Assistant Professor Department of Information Technology, \\ ${ }^{1,2}$ G H Raisoni College of Engineering, Nagpur, India
}

\section{ABSTRACT}

In this paper Secondary network requirement frequency scanning is implemented to show new scanning methodology. The Min-Max algorithm is to formulation of a fair channel allocation is the maximum interference occur in the network transmission link reduce to a minimize value is shown by using Energy detection, Cognitive scanning, a special CYWM6935 cypress scanner module. The main reason behind spectrum sensing is the detection of vacant channel in the scanned spectrum. Energy detection does not require any information regarding primary signal. Thus it is used for both time and frequency domain. The use of CYWM6935 is used to display the $2.4 \mathrm{GHz}$ of ISM band in the range of 50 meter, where detected signal will then display in the LCD. Here design used RSSI, SSID, MAC Address maximum and achievable data rate, security is major ways to test. In this paper new modified type of scanning is also introduced in comparison to dynamic \&t initial scanning which gives the more precision in detection white space in spectrum. A cognitive scanning is used to improve the methodology by optimizing antenna, Tran's-receiver, controller unit, switching unit \&t special power supply is implemented. A comparison to RSSI level is used to make decision for free to use, busy with week connectivity, busy with strong connectivity is proposed to show signal strength in $\mathrm{dB}$. At final implementation cypress module is used to scan for Urban, Rural \& for other specific application mode is deployed to scan for $2.4 \mathrm{GHz}$ band. Depending on these results are shown for advance scanning for 1 \& 6 channel which was always a dominating channel out of 14 channels in Wi-Fi.

\section{KEY WORDS: CYPRESS MODULE, RSSI, MIN-MAX, ISM, COGNITIVE, ENERGY DETECTION.}

\section{INTRODUCTION}

In the research part design Femtocell is required to utilize a free communicating channel by client, these channel are available in network which is actually dynamically given to client to used promptly ch no. 1,6 \&t 11 only. In normal there are total 14 channel are there to utilize. Due

\section{ARTICLE INFORMATION}

*Corresponding Author: nikhil.wyawahare@raisoni.net Received 17th Oct 2020 Accepted after revision 25th Dec 2020 Print ISSN: 0974-6455 Online ISSN: 2321-4007 CODEN: BBRCBA

Thomson Reuters ISI Web of Science Clarivate Analytics USA and Crossref Indexed Journal

\section{1) Clarivate rossef}

NAAS Journal Score 2020 (4.31)

A Society of Science and Nature Publication,

Bhopal India 2020. All rights reserved.

Online Contents Available at: http//www.bbrc.in/

Doi: $h t t p: / / d x$.doi.org/10.21786/bbrc/13.14/42 to effect of co-channel interference these channels are mostly preferred as shown in figure $3.12 .4 \mathrm{GHz}$ channel and its co- channels availability, [Jaeweon Kim, IEEE2010, John Sydor, David Roberts, Bernard Doray and Amir Ghasemi, IEEE INFOCOM 2011] how these are separated with respect to channel centre frequency with $22 \mathrm{MHz}$ width. In research observation channel no 14 i.e. 2.484 $\mathrm{GHz}$ frequency typically available at last and rarely it's been used if all channels are busy at same time.

Which reflects the $99 \%$ of channels utilization which is not possible through access point/router in any network deployment. If a corrective solution is designed also by any router only channels 1,6 \&t 11 used by any way! Thus eventually 2, 3, 4, 7, 8, 9, 10, 12, 13 are getting affected due to these three. Looking toward this approach [Farzad Hessar, IEEE-2013 \&t Kazushi Muraoka Hiroto

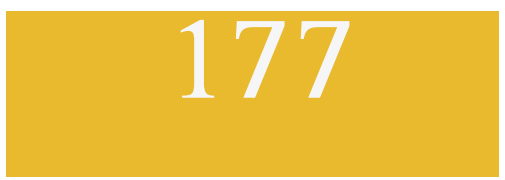


Sugahara Masayuki Ariyoshi, IEEE-2011] “Design and Implementation of Configurable Femtocell base on White space" is need to design to utilize not only for already established network but also for the new external network created Femtocell. In this case of design Femtocell is working in poor signal strength reception location. Adequate channels to communicate with easy sharing of data between all clients. [Nikhil P. Wyawahare, Dr. S. L. Haridas, Helix-2019] This is possible if cognitive scanning of channels are done through external source and finding out which channels are busy-partial busyfree as per load, RSSI strength, offset frequency like parameter observation which is not available in normal access point.

Implementation: Fig.1 illustrated Channel allocation is defined as allocation of channel of frequency spectrum in such a way so that all the users which are keen to carry out their communication through the spectrum will be proficient to transmission and reception of data without having interference with each other. [Wyawahare NP, Haridas SL, IC3I -2014, Nikhil P. Wyawahare, S. L. Haridas, IJST-2017 \& Rizwan Musani, Nikhil P.Wyawahare, IEEE2015] The following points to allocate channels are taken into account by Min-Max algorithm. Allocations of the resources are done on the basis of demand. The objective of this algorithm is formulation of a fair channel allocation is the maximum interference occur in the network transmission link reduce to a minimize value. This algorithm is used for allocation of channel and to use data given by the RF module which is used to sense the spectrum. Min-Max algorithm firstly divides the frequency spectrum in suitable number of division. Further then frequency division of above divided spectrum is done into appropriate number of divisions. After that the phase and magnitude of the scanned spectrum is calculated.

Figure 1: Min Max Algorithm

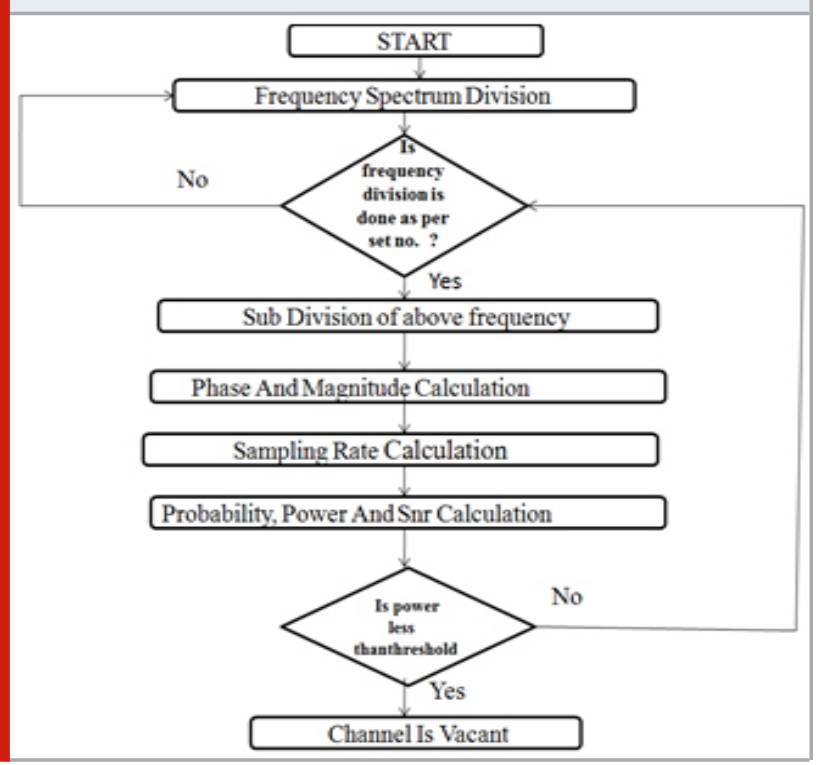

Figure 2: Flow Chart of Tx -Rx Over Serial Port

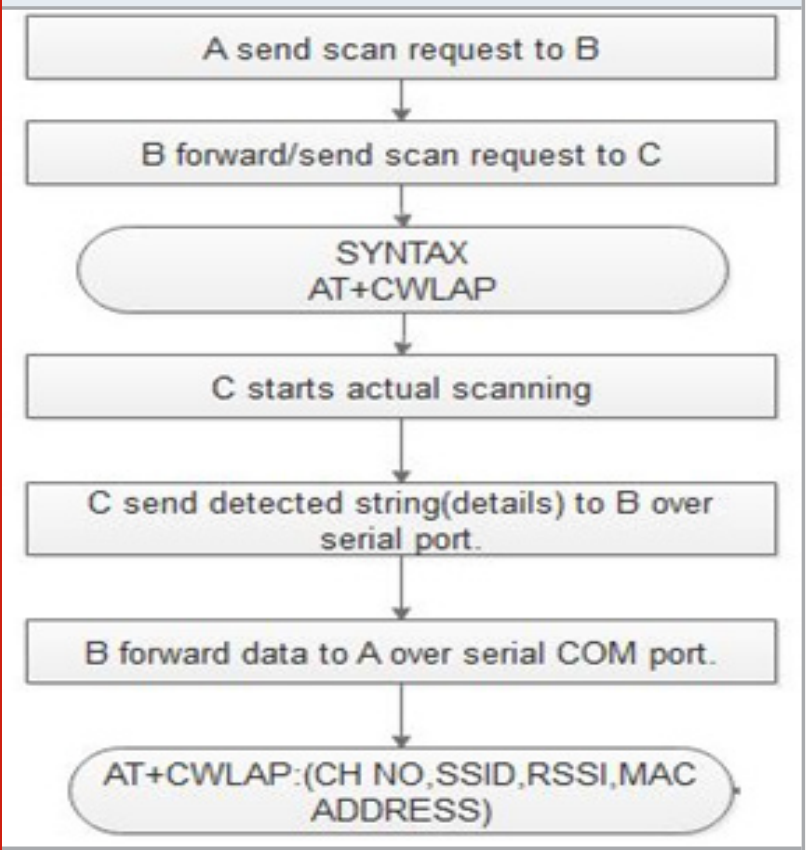

Figure 3: Modes of Scanning

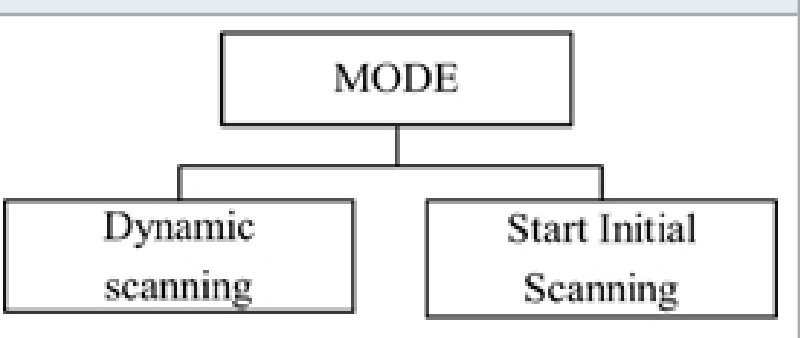

PART A: Energy Detection Method: For white space based application devices the main task is spectrum sensing. The spectrum sensing is the continuous scanning of frequency spectrum. The main reason behind spectrum sensing is the detection of vacant channel in the scanned spectrum. The spectrum should be used by secondary users in such a way that there will be no interference among the primary and secondary users. Energy detection method is the method used here for the spectrum scanning. This method does not require any information regarding primary signal. [Rohit Nandanwar, Nikhil P.Wyawahare, ICESS-2016 \& Nikhil P. Wyawahare, Dr. S. L. Haridas, IJCA-2017] This method can be used for both time and frequency domain. In Scanner design all parameters like SSID, RSSI \& MAC address are going too measured. Using Wi-Fi scanner all 14 channels of $2.4 \mathrm{GHz}$ band is scanned. Scanner is attached with access point to serve the service in maximum allocated distance and no of users. Fig. 3 illustrated modified scanning mode for the cognitive white-fi scanner, in comparison to Active and passive scanning. It is specially design for Femtocell network to seek ideal channel for communication by initial scanning \& dynamic scanning. 
Initial scanning is done at initial stage, when scanner starts scanning. [Shyamali Amale, Prof. Nikhil Wyawahare, IJIRCC-2017] At initial stage it will start scanning number of free channels and number of busy channels and go for free channel to utilize. Et in Dynamic it scans continuously and generate result for busy channels as well as free channels. Even after getting free channel to utilize it will start scanning for best vacant channel to utilize.

\section{PART B: Cognitive Scanning System}

\section{RESULTS AND DISCUSSION}

Figure 4: Cognitive Radio scanning Antenna Unit

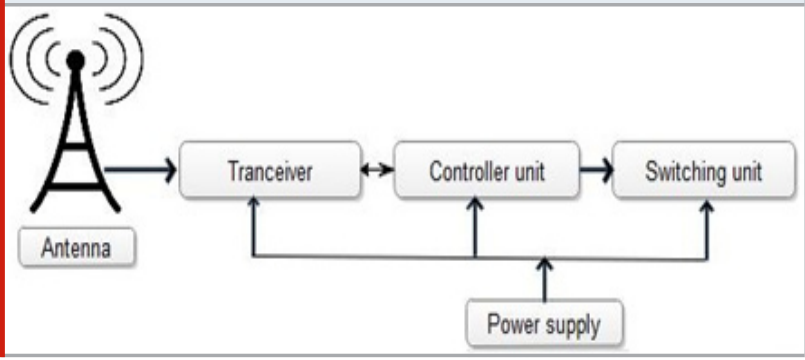

Figure 5: Advance scanning result of spectrum for channel 1 \&t 6

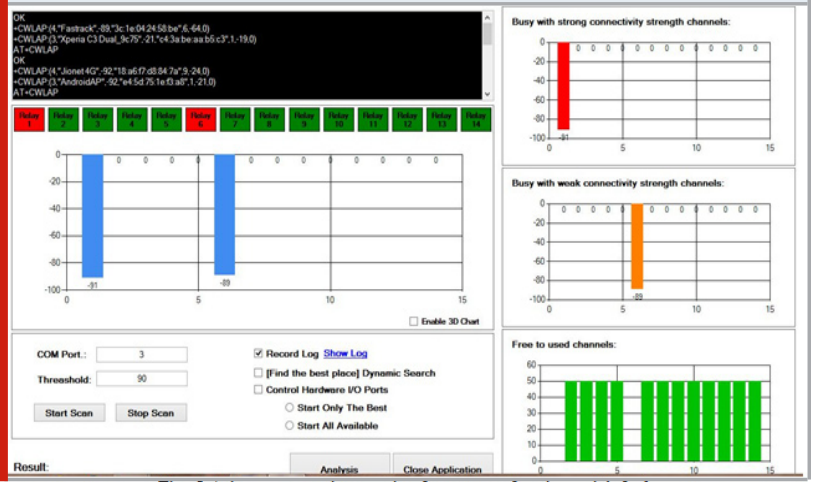

Scanning result for 14-channel number 1, 2, 6 and 11 are busy and remaining 10 channels are free. Result showing received signal strength for each device, E.g. RGI which present on channel number 2 with strength -86 and Mac address "e3de272b1fd5" Fig. 6 illustrated graphical representation for SSID "Mahima", strength vs count. Here Strength for SSID mahima varies between $-88 \mathrm{~dB}$ to $-83 \mathrm{~dB}$ thus above graphs are plotted by taking interval as 10.Graph plotted by taking frequency as parameter, for selected SSID graph is generated by taking interval 8.Above graph is result for offset frequency which fluctuates between negative and positive scale. Graph is generated for SSID "mahima" by taking interval of 8 and plotting graph for channel Vs count is as shown in above diagram. Where, count represents value of repetition result for selected SSID and channel is use to show channel number which was used by that device. Fig.7 illustrating the Femtocell having the very strong reception of signal RSSI at $-10 \mathrm{~dB}$ to $-13 \mathrm{~dB}$ in range.
Figure 6: Graphical plotting for SSID vs. Strength Parameter Ct SSID vs Frequency Parameter

\begin{tabular}{|c|c|c|c|c|c|}
\hline \multicolumn{2}{|c|}{ 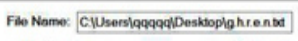 } & \multicolumn{3}{|l|}{ Bromee Log Filo } & \multirow{2}{*}{$\begin{array}{c}\text { Close } \\
\text { Plot Groph }\end{array}$} \\
\hline ssiv: maki & $\checkmark$ Porat & r. Svength & Intervot: [ & 10 & \\
\hline
\end{tabular}
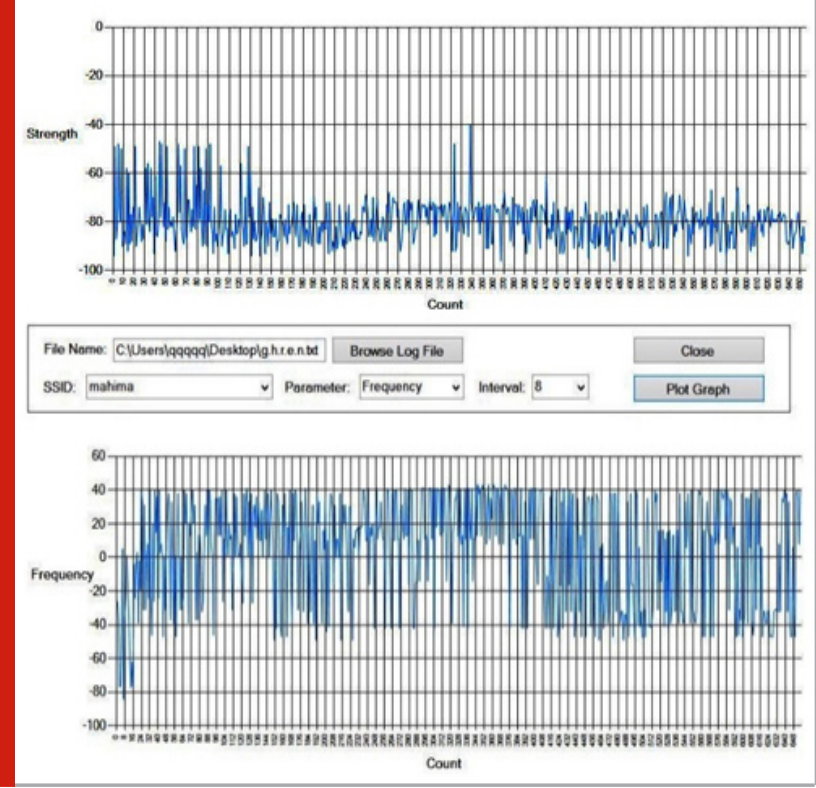

Figure 7: Graph for channel Vs count for selected SSID
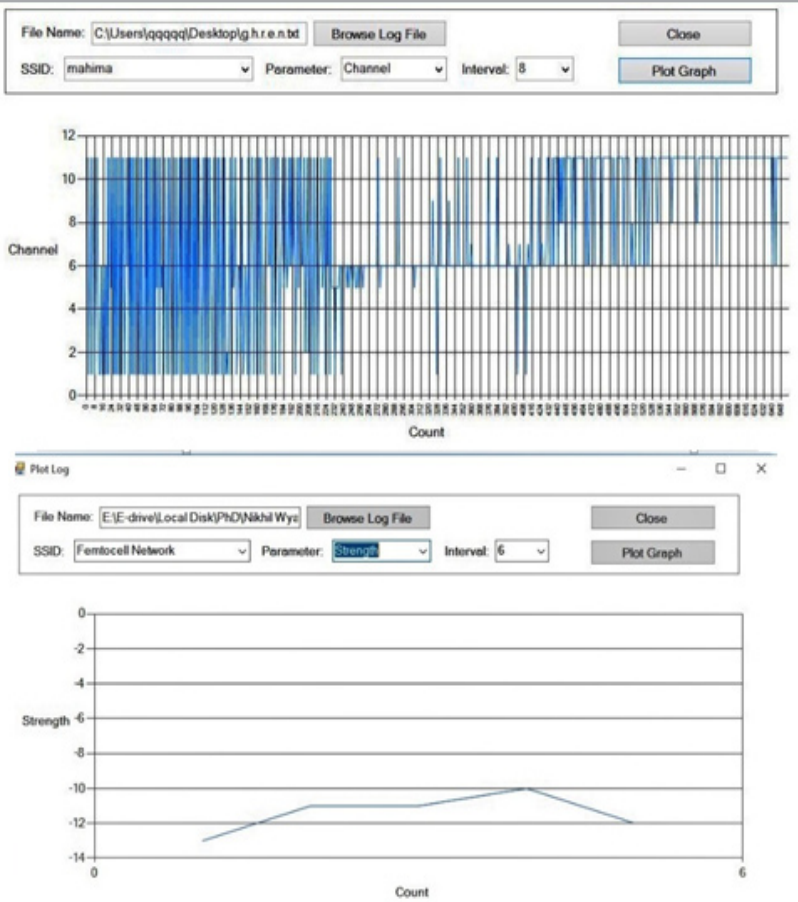

Fig.8 illustrating the graphical representation to have Femtocell SSID scanning in offset frequency VS Count. Offset frequency is representing the adjustment in the frequency component over the co channel interference problem. Channel no 10 is frequently used by the SU network to provide services through base station and allotment of channel through switching relay section. 
Figure 8: Graphical Plotting of Femtocell SSID Frequency VS Count

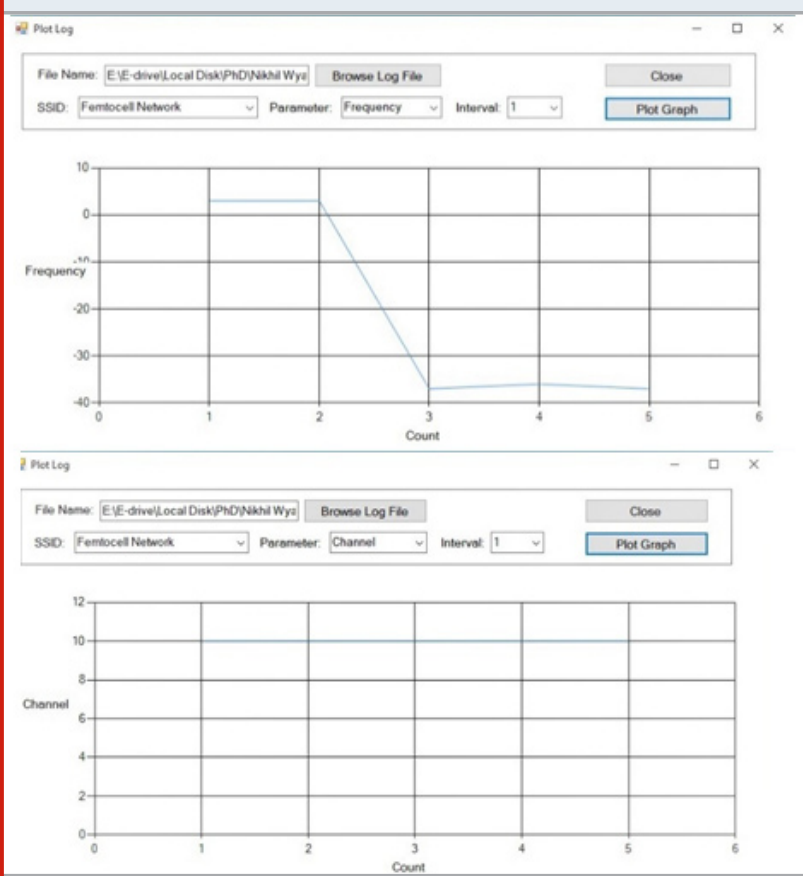

\section{CONCLUSION}

Scanning is done with feature detection technology to find channel band in 2.412 to $2.248 \mathrm{GHz}$. Over to this type of controller is required to take decision faster as to release channel relay for call establishment in Secondary network. Following results are oriented in two main time slot of testing as illustrated in fig. 5 Due to this change any signal change at any moment will be easily detected and scanned as per certain parameter like, RSSI, Offset frequency, channel no likewise. Feature detection of Min-Max algorithm which is implanted in improved scanner module working as "Cognitive White- Fi scanner" or "Hot- Spot" i.e. Femtocell base station server to be facilitated with the appropriate spectrum channel utilization. At final scanner is initial scan to search actively, on later its change to dynamic scanning.

\section{REFERENCES}

Analysis of the FCC's Positions, Policies and Comments on Secondary Markets http: //Principles for Promoting the Efficient Use of Spectrum by Encouraging the Development of Secondary Markets, Policy Statement (FCC 00-401).

Farzad Hessar, Student Member, IEEE, Sumit Roy, Fellow, IEEE, "Capacity Considerations for Secondary Networks in TV White Space”arXiv:1304.1785v1 [cs.NI] 28 Mar 2013.
Jaeweon Kim, Student Member, IEEE, and Jeffrey G. Andrews, Senior Member, IEEE, "Sensitive White Space Detection with Spectral Covariance Sensing” in IEEE TRANSACTIONS ON WIRELESS COMMUNICATIONS, ACCEPTED FOR PUBLICATION, 2010.

John Sydor, David Roberts, Bernard Doray and Amir Ghasemi, "A Generic Cognitive Radio Based on Commodity Hardware", in IEEE INFOCOM 2011 Workshop On Cognitive \& Cooperative Networks Kazushi Muraoka Hiroto Sugahara Masayuki Ariyoshi, "Monitoring-Based Spectrum Management for Expanding Opportunities of White Space Utilization", in 2011 IEEE International Symposium on Dynamic Spectrum Access Networks (DySPAN).

Nikhil P. Wyawahare, Dr. S. L. Haridas "Adaptive White-fi Spectrum Channel Deployment Intended to Configurable Femtocell Network" Helix Vol. 9 (3): 49744980 DOI 10.29042/2019-4974-4980 ^ 2019 The Author (s); Helix E-ISSN: 2319-592; P-ISSN: 2277-3495.

Nikhil P. Wyawahare, Dr. S. L. Haridas "Conniving challenges in Secondary Femtocell Network using DSA on White-Space channel" International Journal of Computer Applications (0975 - 8887) Volume 169 No.7, July 2017.

Nikhil P. Wyawahare, S. L. Haridas, "A Novel OSA based ISM Band Channel Scanner Design for Identification and Deployment of White-Spaces" Indian Journal of Science and Technology, Vol 10(43), DOI: 10.17485/ ijst/2017/v10i43/115338, November 2017, ISSN (Print): 0974-6846, ISSN (Online): 0974-5645

Rizwan Musani, Nikhil P.Wyawahare International Conference on "Design and Implementation of Feature Detection Based Unused Spectrum Sensing: ISM Band" IEEE 2nd International Conference on Knowledge Collaboration in Engineering March 27- 28, 2015 9781-4799-8619-4/15/\$31.00 @2015 IEEE.

Rohit Nandanwar, Nikhil P.Wyawahare "Comparison \& Detection of Power/SNR ratio for Scanned White Spaces over ISM Band: A Review” IEEE Sponsored 3rd International Conference on Electronics And communication System (ICESS 2016), 978-1-46737832-1/1/16/\$31.00, 2016

Shyamali Amale, Prof. Nikhil Wyawahare " Analysis of scanned channel estimation in 2.4 GHz ISM Band" International Journal of Innovation Research in Computer and Communication Engineering,(IJIRCC) Vol, Issue1, January 2017, ISSN 2320-9801.

Wyawahare NP, Haridas SL. Designing challenges to utilize white-space for configurable femtocell base on slotted TDM. IEEE, International Conference on Contemporary Computing and Informatics (IC3I); 2014. p. 1076-80. Crossref. 